\title{
Lohnpolitik und Beschäftigung - Debatte ohne Ende?
}

\author{
von Jürgen Jerger und Oliver Landmann \\ Universität Duisburg und Universität Freiburg im Breisgau
}

Revidierte Fassung: Juni 2001

\begin{abstract}
This paper takes a fresh look at the often confused - and therefore confusing - debate on the role of wage policy in tackling Germany's unemployment problem. The key issues in this debate are the relative importance of wages and aggregate demand in the determination of employment, the appropriate behavior of wages in relation to productivity growth, and the significance of the purchasing power effect of wages. We argue that the most useful tool to address and resolve these issues is the basic textbook model of aggregate supply and aggregate demand. Most importantly, the model predicts that employment is governed by the ratio of aggregate nominal demand and the nominal wage level. The empirical picture strongly supports this assertion. In contrast, the relation between the rate of wage growth and the rate of productivity growth does not play the causal role in determining employment that is widely attributed to it.
\end{abstract}

PD Dr. Jürgen Jerger

Universität Duisburg

Lotharstr. 65

D-47057 Duisburg

Tel.: ++49(0)203-379-2354

Fax: ++49(0) 203-379-1844

Email: erger@uni-duisburg.de
Prof. Dr. Oliver Landmann

Universität Freiburg

Platz der Alten Synagoge

D-79085 Freiburg

Tel.: $++49(0) 761-203-2326$

Fax: ++49(0)761-203-2405

Email: landmann@ vwl.uni-freiburg.de 


\title{
Lohnpolitik und Beschäftigung - Debatte ohne Ende?
}

\author{
von Jürgen Jerger und Oliver Landmann
}

\section{Eine polarisierte und konfuse Debatte}

Kaum ein Thema der wirtschaftspolitischen Debatte in Deutschland ist so alt, lebt mit solcher Regelmäßigkeit immer wieder auf und provoziert so hitzige Auseinandersetzungen wie die Auswirkungen der Lohnpolitik auf die Beschäftigung. Dass sich die Tarifvertragsparteien, für die im wiederkehrenden Verhandlungspoker vitale Verteilungsinteressen auf dem Spiel stehen, auch im Kampf um die öffentliche Meinung nichts schenken, ist nicht weiter verwunderlich und wird sich wohl auch nicht so rasch ändern. Arbeitgeber werden immer mit ihrer Kostenbelastung argumentieren und die Gefahren beschwören, die den Arbeitsplätzen von überhöhten Tarifabschlüssen drohen. Die Gewerkschaften ihrerseits werden weiterhin ihren Anteil am volkswirtschaftlichen Kuchen einfordern und die Frage stellen, wie denn Arbeitsplätze erhalten, geschweige denn neu geschaffen werden sollen, wenn die Kaufkraft der Arbeitnehmer mit dem wachsenden Produktionspotential der Wirtschaft nicht mindestens Schritt hält. In diesem Sinne ist unsere Titelfrage schnell beantwortet: Ein Ende der Debatte ist nicht abzusehen.

Schon eher mag sich eine breitere Öffentlichkeit darüber wundern, dass auch der wissenschaftliche Sachverstand - so weit er sich wahrnehmbar in die Debatte einschaltet - ein Bild der Zerstrittenheit bietet. Von außen ist dabei nicht ohne weiteres zu erkennen, ob dies daran liegt, dass die Zusammenhänge zwischen Lohnpolitik und Beschäftigung wissenschaftlich ganz einfach noch nicht geklärt sind, oder daran, dass es mächtige Interessen verstehen, "Experten" für ihre Sache zu instrumentalisieren, oder vielleicht auch nur daran, dass es vereinfachende und polarisierende Positionen in der Arena der öffentlichen Meinungsbildung besonders leicht haben, das Scheinwerferlicht auf sich zu ziehen. Wir werden im folgenden argumentieren, dass die in Deutschland gleichsam zum Ritual gewordene lohnpolitische Debatte der Öffentlichkeit ein stark verzerrtes Bild davon vermittelt, was die Makroökonomik über die Wirkungen der Lohnpolitik tatsächlich zu sagen vermag.

Wie weit die Meinungen bisweilen auseinandergehen, hat zuletzt mit aller Deutlichkeit das Jahresgutachten 1999/2000 des Sachverständigenrates zur Begutachtung der gesamtwirtschaftlichen Entwicklung gezeigt, in dem sich sowohl die Ratsmehrheit als auch ein Minderheitsvotum des Ratsmitglieds Jürgen Kromphardt ausführlich damit befassen, "wie wir der Vollbeschäftigung wieder näher kommen können" (Ziffer 327ff.). Zur Rolle, die hierbei die Lohnpolitik zu spielen hat, wird der staunende Leser unter anderem mit folgenden Auffassungen konfrontiert:

\footnotetext{
${ }^{1}$ Universität Duisburg bzw. Universität Freiburg i.Br. Einem anonymen Referee danken wir für wertvolle Verbesserungsvorschläge.
} 
"Zwischen Lohnniveau und Beschäftigung besteht ein enger Zusammenhang." (Ratsmehrheit, Ziffer 332)

"Die Lohnpolitik kann keinen Beitrag zur Erhöhung der Beschäftigung leisten." (Minderheitsvotum, Ziffer 366)

"Als Faustregel muss gelten: Die Tariflohnanhebungen werden so lange unterhalb der - bereinigten - Fortschrittsrate der Arbeitsproduktivität gehalten, bis Vollbeschäftigung erreicht ist." (Ratsmehrheit, Ziffer 335)

"Steigen die Reallöhne langsamer als die Produktivität, so führt der damit induzierte Nachfrageausfall auf den Gütermärkten zu einer Gefährdung der Beschäftigung.” (Minderheitsvotum, Ziffer 366)

"Das Argument, dass ein Nachfrageausfall zu verzeichnen sei, wenn die Tariflohnanhebung bei hoher Arbeitslosigkeit unterhalb des Produktivitätsfortschritts gehalten wird, und dass deshalb eine solche Tarifpolitik im Hinblick auf mehr Beschäftigung nicht verfolgt werden sollte, ist falsch.” (Ratsmehrheit, Ziffer 335)

"Der Anstoß zu mehr Beschäftigung kann nicht von der Lohnpolitik kommen, sondern nur von den Gütermärkten." (Minderheitsvotum, Ziffer 371)

”Von einer Besserung der Konjunktur darf man ... keine übermäßigen Beschäftigungswirkungen erwarten." (Ratsmehrheit, Ziffer 337)

Wem ist angesichts dieser Widersprüche der Schluss zu verübeln, dass die Wissenschaft auf die einfachsten und grundlegendsten Fragen der Lohn- und Beschäftigungspolitik offenbar noch keine klaren Antworten weiß? Wir versuchen im folgenden zu zeigen, warum dies dennoch ein Fehlschluss wäre. Wenn solche Auseinandersetzungen über die Lohnpolitik jeweils mehr Hitze als Licht erzeugen, so liegt dies unter anderem einfach daran, dass in der Debatte die elementaren Denkinstrumente, mit denen Studenten der Volkswirtschaftslehre schon in den ersten Semestern makroökonomische Fragestellungen zu analysieren lernen, offenbar leicht vergessen gehen. Hierunter leidet nicht nur die Kommunikation der Vertreter gegensätzlicher Meinungen untereinander, sondern auch das Bild, das die Makroökonomik als wissenschaftliche Disziplin gegenüber der Öffentlichkeit abgibt.

Wir beginnen daher in Abschnitt 2 mit einer Charakterisierung des elementaren makroökonomischen Angebots-Nachfrage-Modells und zeigen, wie dieses einfache analytische Denkwerkzeug die Beschäftigungswirkungen der Lohnpolitik und der Nachfragepolitik zu klären hilft. Dabei wird sofort deutlich, warum der Streit darüber, ob der Anstoß zu mehr Beschäftigung von der Lohnpolitik oder von den Gütermärkten, und damit von der Nachfragepolitik, zu kommen habe, am Kern des Problems vorbei geht. In Abschnitt 3 illustrieren wir die empirische Relevanz unseres Arguments. Einigkeit scheint in der ganzen Debatte am ehesten noch darüber zu bestehen, dass man die Lohnpolitik zur Arbeitsproduktivität in Beziehung setzen muss, wenn man ihre Wirkungen analysiert. In Abschnitt 4 gehen wir daher auf die vielzitierte Produktivitätsregel der Lohnpolitik ein und erläutern, warum eine Gegenüberstellung von Reallohnanstieg und Produktivitätsfortschritt keine einfachen Rückschlüsse auf das Verhalten der Lohnpolitik zulässt. In Abschnitt 5 greifen wir die vieldiskutierte "Kaufkrafttheorie" des Lohnes auf und zeigen, dass sich an den Implikationen des Lehrbuchmodells nichts Grundsätzliches ändert, wenn man die Kaufkraft des Lohneinkommens explizit als Nachfragefaktor berücksichtigt. Ein Fazit ziehen wir schließlich in Abschnitt 6. 


\section{Was sagen die Lehrbücher?}

Wer einen Blick in die Lehrbücher der Makroökonomik wirft, käme nie auf den Gedanken, dass die Beschäftigungswirkungen der Lohnpolitik oder der Nachfragepolitik Anlass zu irgendwelchen Grundsatzdebatten bieten könnten. Wohl hat es die bekannten Kontroversen über technische Fragen wie die optimale Strategie der Nachfragesteuerung, die angemessene Modellierung der Lohnbildung und anderes gegeben. Aber darüber, wie die Auswirkungen von Lohnänderungen und Güternachfrageimpulsen auf Produktion, Beschäftigung und Preise grundsätzlich zu analysieren sind, herrscht heute doch ein recht breiter Konsens. Kaum ein Lehrbuch, das nicht das Modell der aggregierten Nachfrage und des aggregierten Angebots das sog. AS-AD-Modell - als "Arbeitspferd" für die Beschreibung dieser Zusammenhänge einsetzt. Als repräsentativ kann gelten, was ein prominenter Lehrbuchautor über das AS-ADModell sagt: "When confronted with macroeconomic questions, it is the model that I typically use to organize my thoughts."

Die wichtigsten Elemente dieses Modells lassen sich ohne größeren formalen Aufwand mit Hilfe von Abbildung 1 erläutern. ${ }^{3}$ Die im Preis-Output-Quadranten als steigende Funktion eingezeichnete Angebotsfunktion stellt das Verhalten der Güterproduzenten dar. Wenn man davon ausgeht, dass die Anbieter auf den Gütermärkten typischerweise unter Bedingungen des unvollkommenen Wettbewerbs operieren, ist es am zweckmäßigsten, die Angebotsfunktion ungeachtet ihres Namens - als Abbild des unternehmerischen Preissetzungsverhaltens zu interpretieren. Die zugrundeliegende Vorstellung ist, dass die Unternehmen ihre Preise mit einem Aufschlag auf ihre Grenzkosten kalkulieren. Je mehr Wettbewerbsdruck auf den Gütermärkten herrscht, desto geringer ist dieser Aufschlag. Die Grenzkosten reflektieren hauptsächlich das Verhältnis zwischen dem Nominallohnniveau und der Grenzproduktivität der Arbeit, wobei letztere wiederum vom Stand der Technologie sowie von der Kapitalintensität der Produktion abhängt.

Kosten- und damit auch preistreibend wirken somit vor allem Nominallohnerhöhungen, wenn sie über die Produktivitätsfortschritte hinausgehen, die sich durch den Ausbau des Kapitalstocks und den technischen Fortschritt einstellen. Jeder derartige Kostenschub lässt sich, wie in Abbildung 1 angegeben, durch eine Verlagerung der aggregierten Angebotsfunktion nach oben darstellen. Demgegenüber bringt der steigende Verlauf der Angebotsfunktion zum Ausdruck, dass eine Ausweitung der Produktion selbst bei konstanten Nominallöhnen und unverändertem Produktionspotential der Volkswirtschaft zu steigenden Preisen führen kann, weil die Unternehmen gegen ihre Kapazitätsgrenzen stoßen und hierdurch Arbeitsplätze mit immer geringerer Grenzproduktivität und entsprechend höheren Grenzkosten entstehen.

Abbildung 1 etwa hier

\footnotetext{
2 Blanchard (2000a), S. 125. Das Ausmaß des Konsenses wurde auch durch das Symposion "Teaching Macroeconomic Principles" an der Jahrestagung 2000 der American Economic Association verdeutlicht (American Economic Review, P.P., Vol. 90, No. 2, 2000, 76-94).

3 In Jerger/Landmann (2001) sind diese Zusammenhänge etwas formaler beschrieben.
} 
Die aggregierte Nachfragefunktion besagt, dass die effektive Güternachfrage zurückgeht, wenn das Preisniveau steigt. Im einfachsten Fall kommt dieser Zusammenhang dadurch zustande, dass ein Anstieg des Preisniveaus bei einer unveränderten Vorgabe für die nominale Geldmenge die reale Geldversorgung der Wirtschaft verringert, was das Geld auf dem Kapitalmarkt verteuert und somit die Ausgabenlust dämpft. Daneben reagiert die Güternachfrage auch auf Impulse, die ihren Ursprung in der Politik (Geld- und Finanzpolitik) oder im privaten Sektor (Nachfrageschocks) haben. Solche Impulse lassen sich durch Verlagerungen der Nachfragefunktion nach rechts (expansive Impulse) bzw. nach links (kontraktive Impulse) darstellen. Die Frage, inwieweit auch von der Lohnpolitik nachfrageseitige Impulse ausgehen könnten, stellen wir bis Abschnitt 5 zurück.

Der Schnittpunkt der beiden Funktionen stellt ein aggregiertes Gütermarktgleichgewicht in dem Sinne dar, dass die Unternehmen einerseits ihren Output zu Preisen anbieten, die für sie optimal sind, und andererseits bei dem hieraus resultierenden Preisniveau die produzierte Gütermenge genau der aggregierten Nachfrage entspricht. Gleichgewicht bedeutet aber nicht unbedingt auch Vollbeschäftigung. Dies wird in Abbildung 1 durch die vertikale Linie verdeutlicht, die das Produktionspotential der vollbeschäftigten Volkswirtschaft markiert. In dem Grade, wie die Kapitalbildung und der technische Fortschritt die Arbeitsproduktivität erhöhen bzw. auch das Arbeitskräftepotenzial zunimmt, verlagert sich im Zeitablauf natürlich auch diese Vollbeschäftigungsgrenze nach außen. $\mathrm{Zu}$ Änderungen des Beschäftigungsgrades kommt es immer dann, wenn sich die Lage des Angebots-Nachfrage-Gleichgewichts im Verhältnis zur Lage der Vollbeschäftigungsgrenze verändert.

Die Lohnpolitik kontrolliert von den zahlreichen Faktoren, die auf die Lage des Gleichgewichts einwirken, einen wichtigen, nämlich das Nominallohnniveau. Damit beeinflusst sie die räumliche Lage der aggregierten Angebotsfunktion. Wesentlich ist in diesem Zusammenhang aber die Einsicht, dass die Kontrolle über die räumliche Lage einer einzelnen Funktion nicht ausreicht, um auch die Lage und damit die Eigenschaften des makroökonomischen Gleichgewichts insgesamt zu kontrollieren. Denn dieses reflektiert ja immer simultan das Verhalten beider Funktionen. Konkret bedeutet dies zum Beispiel, dass die Lohnpolitik mit der Kontrolle der Nominallohnentwicklung nicht auch schon die Reallohnentwicklung - also die Entwicklung der Lohnkaufkraft - kontrolliert, weil letztere davon abhängt, wie sich die Nominallöhne im Verhältnis zum Preisniveau verhalten. Die Tarifparteien mögen zwar mit ihren nominalen Lohnabschlüssen bestimmte reale Ergebnisse anpeilen, aber die Überwälzung der Lohnänderungen auf die Güterpreise entzieht sich ihrem Einfluss. Bedeutsam für die Überwälzungsspielräume sind die Gegebenheiten auf der Nachfrageseite des Gütermarkts, deren Steuerung wiederum der Nachfragepolitik obliegt.

Entsprechendes gilt für die Nachfragepolitik: Weil sie nur auf die Lage der Nachfragefunktion direkt einwirken kann, nicht aber auf die Lage der aggregierten Angebotsfunktion, ist sie, auf sich allein gestellt, nie in der Lage, sowohl den realen Output als auch das Preisniveau zu steuern. Bestenfalls kann sie das Produkt dieser beiden Größen, also die nominale Gesamtnachfrage, steuern. Inwieweit sich jedoch ein gegebenes nominales Wachstum in einem realen Mengenwachstum bzw. in einem Anstieg des Preisniveaus manifestiert, lässt sich nur aus der Interaktion von Angebot und Nachfrage heraus bestimmen. Immerhin bedeutet dies, dass die Wachstumsrate des nominalen Inlandsprodukts als Indikator für das Verhalten der aggregierten Gesamtnachfrage interpretiert werden kann. Wie zweckmäßig dies für die Analyse 
makroökonomischer Angebots-Nachfrage-Zusammenhänge ist, zeigt etwa die exemplarische Diskussion des Modells durch Gordon (2000, Kap. 8).

Die beschäftigungspolitisch entscheidende Implikation unserer Modellbetrachtung lässt sich sehr einfach zusammenfassen: Wenn die Beschäftigung davon abhängt, wo das AngebotsNachfrage-Gleichgewicht im Verhältnis zur Vollbeschäftigungsgrenze liegt, und wenn die Lage des Angebots-Nachfrage-Gleichgewichts davon abhängt, wie sich die Nachfragepolitik und die Lohnpolitik relativ zueinander verhalten, so fällt die Beschäftigung notwendigerweise in die gemeinsame Verantwortung der Lohnpolitik und der Nachfragepolitik. So trivial dieses Ergebnis anmuten mag, so weitreichend ist seine Bedeutung. Denn es zeigt mit aller Deutlichkeit, dass die simplifizierenden, in den beschäftigungspolitischen Debatten so populären Pseudo-Diagnosen - „die Löhne sind zu hoch“ hüben, „es fehlt an Nachfrage“ drüben allesamt zu kurz greifen. Unterbeschäftigung entsteht dadurch, dass die im Hinblick auf die Vollbeschäftigung erforderliche Kooperation der Lohnpolitik und der Nachfragepolitik nicht zustandekommt.

Ein naheliegender Schluss aus diesen Überlegungen ist die Notwendigkeit bzw. Wünschbarkeit einer Koordination von Lohn- und Nachfragepolitik, da letztlich beide gemeinsam über Beschäftigung, Output und Inflation bestimmen. Konkret könnte man sich vorstellen, dass die Nachfragepolitik einen bestimmten (nicht notwendigerweise konstanten) Zielwert für das Wachstum des nominalen Sozialprodukts vorgibt und durchsetzt. Vor diesem Hintergrund könnten dann die Lohnsetzer die Beschäftigungs- (und Preis-) Konsequenzen ihrer Nominallohnpolitik abschätzen. Würden sich die Nominallöhne im Gleichschritt mit dem nominalen Sozialprodukt bewegen, so resultierte eine inflationsfreie Stabilisierung der Beschäftigung. Eine relativ zum Wachstum des nominalen Sozialprodukts zurückhaltendere bzw. aggressivere Lohnpolitik hätte entsprechende Änderungen der Beschäftigung zur Folge. Dieses Koordinations-Szenario kann als „,doppelte nominale Einkommensregel“ (Jerger 1999, S. 216 f.) bezeichnet werden, da sich sowohl die Nachfragepolitik als auch die Lohnpolitik an ihr zu orientieren hätten. Im Unterschied zu einer herkömmlichen Nominaleinkommensregel, wie sie etwa Hall und Mankiw (1994) vorgeschlagen haben, und die einfach eine bestimmte Reaktion der Geldpolitik auf Nachfrage- und Angebotsschocks vorsieht, dient dieses umfassendere Konzept zugleich auch als Signal und als Richtschnur für die Lohnpolitik. Eine Vorgabe für das Wachstum der nominalen Gesamtnachfrage führt im übrigen zu einem sehr ähnlichen Verhalten der Geldpolitik, wie es die gegenwärtig viel diskutierten Strategien der Zins- und Inflationssteuerung implizieren (Taylor 1999, Jensen 1999).

\section{Das empirische Bild spricht eine deutliche Sprache}

Will das aggregierte Angebots-Nachfrage-Modell beschäftigungspolitisch ernst genommen werden, sollten sich die behaupteten Zusammenhänge zwischen Beschäftigung, Güternachfrage und Lohnpolitik auch in den empirischen Daten wiederfinden. Entsprechend den oben

\footnotetext{
4 Neben der in diesem Abschnitt angeführten Evidenz kann auf eine breite und gut abgestützte empirische Literatur verwiesen werden, die auf dem AS-AD-Modell aufbaut. $\mathrm{Zu}$ nennen sind dabei zum einen makroökonometrische Modelle, die - bei allen Unterschieden - praktisch ausnahmslos diesem Modellierungsparadigma verpflichtet sind (vgl. Whitley 1994 für einen Überblick). Auf der anderen Seite basieren aber auch die moderneren, sparsamer parametrisierten Beschreibungen der Kovaration von Mengen und Preisen auf makroökonomischer Ebene auf diesem Modell. Vor allem zu nennen sind hier die sog. Strukturellen Vektorautoreg-
} 
angestellten Überlegungen verwenden wir in Abbildung 2 als Maß dafür, wie sich die Nachfragepolitik und die Lohnpolitik relativ zueinander verhalten, die Differenz zwischen dem nominalen Wachstum der Güternachfrage $(\hat{X})$ und der Änderungsrate der Nominallöhne $(\hat{W})$ und stellen diese Differenz der Änderungsrate der Beschäftigung $(\hat{N})$ gegenüber. ${ }^{5}$ Die Abbildung zeigt den Zusammenhang jeweils für die Bundesrepublik Deutschland und die USA über knapp vier Jahrzehnte hinweg bis in die frühen 60er Jahre zurück. Auf der Basis von Quartalsdaten der OECD sind jeweils die prozentualen Wachstumsraten gegenüber dem gleichen Quartal des Vorjahres ausgewiesen.

\section{Abbildung 2 etwa hier}

$\hat{N}$ ist jeweils als fette Linie, $\hat{X}-\hat{W}$ als etwas dünnere Linie dargestellt. Den Grafiken für Deutschland und die Vereinigten Staaten ist gemeinsam, dass der Zusammenhang - wie durch das Modell impliziert - sehr eng, wenn auch nicht gerade perfekt ist. ${ }^{-}$Zwei Unterschiede zwischen den beiden Charts fallen auf: Während sich in den USA die beiden Zeitreihen nahezu 1:1 entsprechen, ist in Deutschland die Volatilität des Beschäftigungswachstums deutlich geringer als diejenige von $\hat{X}-\hat{W}$. Dies dürfte ein Reflex der bekannten institutionellen und regulatorischen Arbeitsmarktmerkmale sein, die in Deutschland das Anpassungstempo der Beschäftigung (in beide Richtungen) herabsetzen. Der zweite Unterschied betrifft die Tatsache, dass sich die beiden Zeitreihen im Falle Deutschlands viel seltener oberhalb der Nullinie bewegen als im Falle der Vereinigten Staaten, wo die Beschäftigung nur in vereinzelten Rezessionsjahren absolut zurückgegangen ist. Dies ist das vielzitierte amerikanische 'Beschäftigungswunder' der zurückliegenden Jahrzehnte. Somit wird deutlich, dass sich die Implikationen des Angebots-Nachfrage-Modells bezüglich des Zusammenhangs zwischen Beschäftigung, Güternachfrage und Löhnen nicht nur im zeitlichen Längsschnitt, sondern auch über die nationalen Besonderheiten der Arbeitsmärkte hinweg bestätigt finden.

Weiteren Aufschluss über Gemeinsamkeiten und länderspezifische Unterschiede der in Abbildung 2 gezeigten Zusammenhänge gibt eine ökonometrische Analyse, konkret: eine Regression von $\hat{N}$ auf $\hat{X}-\hat{W}$. Diese führen wir mit Jahresdurchschnittswerten durch, um die

ressionsmodelle, die mit und im Gefolge der Pionierarbeiten von Bernanke (1986) und Blanchard/Quah (1989) weite Verbreitung gefunden haben.

5 Genaugenommen haben wir bisher die Abhängigkeit der Beschäftigung von den Nominallöhnen und der nominalen Nachfrage nur qualitativ beschrieben. Die spezifische funktionale Gestalt der Beziehung, welche die Darstellung in Abbildung 2 suggeriert, ist eine Implikation der einfachen formalen Modellierung in Jerger/Landmann (2001).

6 Wir verwenden folgende OECD-Zeitreihen:

Beschäftigung: Anzahl der abhängig Beschäftigten im privaten Sektor Nominale Gesamtnachfrage: Nominales Bruttoinlandsprodukt

Nominallohn: Entlohnung pro abhängig Beschäftigten im privaten Sektor

7 Auf die in Abbildung 2 illustrierte Korrelation hat in mehreren Papieren bereits Lehment aufmerksam gemacht (z.B. Lehment 1999), der das ( $\hat{X}-\hat{W}$ )-Differential jedoch irreführenderweise als "Lohnzurückhaltung" interpretiert und damit die nachfrageseitigen Determinanten dieser Größe ignoriert. 
Problematik der hohen Intra-Jahres-Volatilität von $\hat{X}-\hat{W}$ für Deutschland zu vermindern. Die Trägheit der Beschäftigungsreaktion wird ermittelt, indem neben dem aktuellen Wert der Rechthandvariablen sukzessive weitere Lags addiert wurden, bis diese nicht mehr statistisch signifikant waren. Für Deutschland erwiesen sich neben dem aktuellen Wert auch die Koeffizienten der beiden Vorjahre als signifikant, im Fall der USA reichte ein Lag von nur_einem Jahr. Die nachfolgende Tabelle enthält die geschätzten Koeffizienten und t-Statistiken. ${ }^{\text {Wäh- }}$ rend sich letztere bei den Schätzungen der Einzelkoeffizienten wie üblich auf den Test dieser Koeffizienten gegen Null beziehen, gibt der t-Wert für die Koeffizientensumme den Test dieser Summe auf einen signifikanten Unterschied zu dem Wert Eins an.

\begin{tabular}{|l|c|c|}
\hline & Deutschland & USA \\
\hline Koeffizient (t-Wert) bei Lag & & $0,772(8,567)$ \\
0 & $0,545(5,021)$ & $0,348(3,903)$ \\
1 & $0,519(4,706)$ & - \\
2 & $0,193(1,768)$ & $1,120(1,043)$ \\
Summe & $1,258(1,182)$ & 1,823 \\
\hline Durbin-Watson & 2,078 & 0,733 \\
\hline korr. Bestimmtheitsmaß & 0,621 & \\
\hline
\end{tabular}

Tabelle 1

Da die Koeffizientensumme für beide Länder nicht signifikant von Eins verschieden ist, findet sich die Grundaussage des theoretischen Modells für beide Länder bestätigt. Allerdings erfolgt in den USA der Großteil der Beschäftigungsanpassung an eine Änderung von $\hat{X}-\hat{W}$ innerhalb eines Jahres, während in Deutschland eine länger andauernder Anpassungsprozess ausgelöst wird.

\section{Produktivitätsregel, Beschäftigung und Preisstabilität}

Es mag erstaunen, dass bisher erst am Rande von der Arbeitsproduktivität die Rede war, wird doch der Produktivitätsfortschritt gemeinhin als Schlüsselgröße für die Lohnfindung angesehen. Konkret formuliert die Sachverständigenratsmehrheit (Sachverständigenrat 1999, Ziffer 333 ff.) als Grundregel, dass die Nominallohnanhebungen im Ausmaß des Produktivitätsfortschritts zu halten seien. Hiervon sei aber bloß eine Stabilisierung der Beschäftigung zu erwarten. Eine Steigerung der Beschäftigung sei demgegenüber nur dann zu erreichen, wenn der Lohnzuwachs hinter dem "bereinigten" Produktivitätswachstum zurückbleibe. Die Bereinigung rechnet dabei statistische Produktivitätsgewinne, die sich einstellen, wenn im Zuge eines Beschäftigungsabbaus überwiegend Arbeitsplätze unterdurchschnittlicher Produktivität

\footnotetext{
${ }^{8}$ Die Gleichung für USA wurde mit der gewöhnlichen Kleinstquadrate-Methode geschätzt. Da diese Methode mit den Daten für Deutschland zu Problemen von serieller Korrelation der Schätzresiduen führte, verwendeten wir dafür einen verallgemeinerten Kleinstquadrate-Schätzer mit einer Korrektur für Autokorrelation erster Ordnung.
} 
wegfallen, heraus. Diese Produktivitätsgewinne sollten von der Lohnpolitik nicht als verteilbare Manövriermasse behandelt werden.

Garantiert die Einhaltung dieser „Grundregel“ die Beschäftigungseffekte, welche die Ratsmehrheit für sie in Anspruch nimmt? Die Logik des makroökonomischen Angebots-Nachfrage-Modells zeigt eindeutig: nein. Da die Lohnpolitik nur auf die Lage der aggregierten Angebotsfunktion Einfluss nimmt, ist sie auf Unterstützung seitens der Nachfragepolitik angewiesen, wenn sich bestimmte Beschäftigungsergebnisse einstellen sollen. Eine wohlwollende Interpretation der Mehrheitsposition des Sachverständigenrates wäre, dass sie dies stillschweigend voraussetzt.

Im Diagramm des Angebots-Nachfrage-Modells von Schaubild 1 wirkt sich das bereinigte Produktivitätswachstum, dessen Tempo vom technischen Fortschritt und von der Kapitalbildung bestimmt wird, in doppelter Weise aus: Einerseits verschiebt es die aggregierte Angebotsfunktion nach unten, weil es (bei gegebenen Nominallöhnen) kostensenkend wirkt und damit auch jeden gegebenen Output zu tieferen Preisen zu produzieren erlaubt. Andererseits vergrößert sich aber auch der Vollbeschäftigungsoutput. Denn Produktivitätswachstum bedeutet ja, dass sich mit jedem gegebenen Faktorinput mehr produzieren lässt. Mit anderen Worten: Die Produktion muss mit der Rate des Produktivitätsfortschritts wachsen, um die Beschäftigung auch nur konstant zu halten. Nicht umsonst spricht man in diesem Zusammenhang von der "Beschäftigungsschwelle", die das reale Wirtschaftswachstum überschreiten muss, bevor auch die Beschäftigung zunimmt. Aber die Lohnpolitik kann durch Einhalten der Produktivitätsregel ein solches Wachstum nicht aus dem Hut zaubern. Produktivitätsorientierte Nominallohnabschlüsse können lediglich die Lohnstückkosten stabil halten und so ihren Beitrag zu stabilen Güterpreisen leisten. Ohne nominales Nachfragewachstum kann bei stabilen Preisen jedoch der Output nicht zunehmen - und wenn der Output nicht zunimmt, so bedeutet dies bei anhaltendem Produktivitätswachstum rückläufige Beschäftigung.

Wir können somit festhalten: Lohnzurückhaltung im Sinne der Produktivitätsregel ist für eine Zunahme der Beschäftigung nicht hinreichend. Sie schafft aber die Voraussetzung dafür, dass die Wirtschaft in die Vollbeschäftigung hineinwachsen kann, ohne dass die Güterpreise steigen. Wenn die Geldpolitik de facto und de jure auf die Aufrechterhaltung der Preisniveaustabilität festgelegt ist, bestimmt die Lohnpolitik die Expansionsspielräume, die die Geldpolitik ausschöpfen kann, ohne ihren Stabilitätsauftrag zu verletzen. In diesem Sinne ist ein Zurückbleiben des nominalen Lohnzuwachses hinter dem bereinigten Produktivitätswachstum ein notwendiges Element einer makroökonomischen Strategie, die gleichzeitig die Beschäftigung erhöhen und die Preisstabilität bewahren soll. Aber erfolgreich kann eine solche Strategie nur sein, wenn die Nachfragepolitik die Spielräume nutzt, die ihr die Lohnpolitik schafft.

Ob die Lohnpolitik ihre beschäftigungspolitische Verantwortung wahrnimmt oder nicht, wird oft an der Entwicklung der Reallöhne relativ zum Produktivitätsfortschritt festzumachen versucht. Typischerweise werden dabei Reallohnzuwächse, die hinter dem Produktivitätswachstum zurückbleiben, als Ausdruck einer "Politik der Lohnzurückhaltung" interpretiert. "Wwar haben wir gesehen, dass in der Lohnpolitik nur über Nominallöhne entschieden werden kann, während sich die Reallöhne unter dem Einfluss angebots- wie nachfrageseitiger Einflüsse en-

\footnotetext{
9 Auf eventuelle nachfrageseitige Wirkungen der Lohnpolitik kommen wir in Abschnitt 5 zurück.

${ }^{10}$ Vgl. z.B. Kromphardt (1999) und Heise/Schulten (1999).
} 
dogen bilden und - wie vor allem Solow (1986) betont hat - allein schon aus diesem Grund nicht als exogene Ursache eines Beschäftigungsproblems in Frage kommen.

Vor dem Hintergrund der lohnpolitischen Diskussionen drängt sich aber dennoch die Frage auf, welchen Informationsgehalt das Verhältnis zwischen dem Reallohnniveau und der Arbeitsproduktivität - oder was dasselbe ist: die Lohnquote - hinsichtlich der Lohnpolitik aufweist. Von einer gewissen Brisanz ist diese Frage vor allem deshalb, weil die Lohnquote in der Bundesrepublik Deutschland wie auch in anderen Volkswirtschaften Kontinentaleuropas seit etwa 1980 substanziell zurückgegangen ist, während gleichzeitig die Beschäftigung stagniert und die Arbeitslosigkeit zugenommen hat. Wenn nun die Lohnquote ein zuverlässiger Indikator für das Verhalten der Lohnpolitik wäre, könnte die Entwicklung seit 1980 den Schluss nahelegen, dass die Lohnpolitik ihren Beitrag zu einer Verbesserung der Beschäftigungslage doch wohl geleistet habe und die Ursachen der gestiegenen Arbeitslosigkeit mithin an anderer Stelle zu suchen seien, also etwa bei einer ungenügenden Expansion der Güternachfrage.

Aber die Lohnquote ist kein zuverlässiger Indikator für das Verhalten der Lohnpolitik. Wie wir an anderer Stelle näher ausgeführt haben (Jerger/Landmann 2001), können Änderungen der Lohnquote entweder auf technologische Entwicklungen zurückgeführt werden, durch die sich die Produktionselastizität des Faktors Arbeit verändert, oder auf Verschiebungen in der Marktmacht von Anbietern und Nachfragern auf den Güter- und Arbeitsmärkten, die einen Keil zwischen den Reallohn und die Grenzproduktivität der Arbeit treiben. Es liegt mittlerweile eine Reihe von theoretischen und empirischen Untersuchungen vor, welche die Koinzidenz von rückläufigen Lohnquoten und steigender Arbeitslosigkeit unter Bezugnahme auf genau diese Faktoren analysieren.

Damit soll nicht behauptet werden, dass von der Lohnpolitik überhaupt keine Einflüsse auf die Lohnquote ausgehen. Kurzfristig müssen abrupte Lohnänderungen allein deshalb schon zu gleichgerichteten Änderungen der Lohnquote führen, weil die Unternehmungen ihre Aktionsparameter Preis, Beschäftigung und Kapitalstock nur mit Verzögerungen (unterschiedlicher Länge) anpassen können und die Möglichkeiten der Faktorsubstitution kurzfristig begrenzt sind. Der Anstieg der Lohnquoten vieler europäischer Länder in den 70er Jahren lässt sich auf diese Weise erklären, allerdings nur als Übergangsphänomen - und um mehr handelte es sich ja auch nicht (Blanchard 2000b, Landmann/Jerger 1993). Denn je weiter die Anpassungs- und Substitutionsprozesse fortschritten, desto mehr erholten sich die Profitraten und verflüchtigten sich entsprechend auch die Verteilungsgewinne der Arbeitnehmer wieder. Was dem oberflächlichen Beobachter dabei als „Lohnzurückhaltung“ vorkommen mochte, war einfach die allmählich einsetzende Wirkung der Anpassungsprozesse, mit denen die Unternehmungen auf den erhöhten Lohndruck reagierten.

Somit zeigt sich: Eine Lohnpolitik, die auf eine Umverteilung des Volkseinkommens von Kapital zu Arbeit angelegt ist, kann nur kurzfristig erfolgreich sein - und auch dies nur auf Kosten der Beschäftigung. Langfristig bleiben zwar die negativen Beschäftigungseffekte erhalten, nicht aber die Erhöhung der Lohnquote. ${ }^{2}$ Im Gegenteil, wenn das Kapital in Reaktion auf den Expropriationsversuch der Gewerkschaften in weniger arbeitsintensive Technologien

\footnotetext{
${ }^{11}$ Vgl. insbesondere Blanchard (1997, 2000b) sowie Blanchard/Giavazzi (2001).

${ }^{12}$ Vgl. Landmann/Jerger (1993) für eine ausführlichere theoretische Begründung und eine empirische Illustration am Beispiel der Bundesrepublik Deutschland.
} 
ausweicht oder arbeitsintensive Produktionszweige in andere Länder verlagert, kann es leicht passieren, dass der Schuss auf längere Sicht nicht nur beschäftigungspolitisch, sondern auch verteilungspolitisch nach hinten losgeht (Caballero/Hammour 1998).

\section{Ein Einwand für Fortgeschrittene: das Kaufkraftargument}

Der Beitrag, den eine zurückhaltende Lohnpolitik zur Erhöhung der Beschäftigung leisten muss, wird, wie wir eingangs gesehen haben, immer wieder mit dem Argument bestritten, dass die Löhne nicht nur Kosten, sondern auch Kaufkraft bedeuten und damit eine wichtige Determinante der gesamtwirtschaftlichen Güternachfrage sind. Wenn Lohnerhöhungen die Güternachfrage beleben - so wird bisweilen gefolgert - müssen sie sich auch positiv auf die Beschäftigung auswirken. Charakteristisch für diese Argumentationsweise ist die folgende Grafik, mit der die IG Metall ihre Mitglieder über die Wirkung der Löhne informiert: ${ }^{\text {L3 }}$

Abbildung 3 etwa hier

Das Argument weist eine lange Geschichte auf, die wenigstens bis auf Jacob Marschak (1927) zurückverfolgt werden kann, aber auch Lerner (1951) und Kalecki (1971) argumentierten entlang der in Abbildung 3 gezeigten Linien. Als Kronzeuge wird aber meist Keynes (1936) zitiert, der in dem berühmten Kapitel 19 seiner General Theory verschiedene Wirkungskanäle diskutiert hat, über die Nominallohnsenkungen das makroökonomische Gleichgewicht und damit auch den Beschäftigungsgrad einer Volkswirtschaft beeinflussen können. Einer dieser Wirkungskanäle war auch der Kaufkrafteffekt. Aus der Summe der von ihm betrachteten Effekte zog Keynes den Schluss, dass die Vollbeschäftigung durch Nominallohnanpassungen allein nicht gewährleistet werden könne. Am besten sei vielmehr eine Politik der stabilen Nominallöhne, durch die sich die destabilisierenden Wirkungen eines volatilen Preisniveaus vermeiden ließen. ${ }^{14}$ Dies impliziert im Einklang mit unseren obigen Überlegungen eine zentrale Mitverantwortung der Nachfragepolitik für die Beschäftigung. An keiner Stelle findet sich bei Keynes jedoch eine Stütze für das Argument, dass Lohnerhöhungen Beschäftigungsgewinne brächten.

Das Argument in Abbildung 3 illustriert anschaulich, was herauskommen kann, wenn man die Wirkungsanalyse eines von außen kommenden Anstoßes unter Ausblendung aller weiteren interdependenten Folgewirkungen auf einen einzigen kurzen Wirkungskanal reduziert. Hierfür hat von Weizsäcker (2000, S. 37 ff.) unlängst in dieser Zeitschrift den Begriff "verkürztes Denken" geprägt. Was die in Abbildung 3 skizzierte Kausalkette ausblendet, ist die Überwälzung der steigenden Löhne auf die Güterpreise und die davon ausgehenden

\footnotetext{
${ }^{13}$ Die Grafik ist als 98_2403.pdf unter der Adresse http://www.igmetall.de/daten_fakten_grafiken/direkt/einkommen/index.html verfügbar (14.2.2001).

${ }^{14}$ Da Keynes noch nicht in Kategorien einer wachsenden Wirtschaft dachte, nahm er bei dieser Schlussfolgerung nicht auf den Produktivitätsfortschritt Bezug. Eine ausführlichere Auseinandersetzung mit dem Erbe des 19. Kapitels der General Theory aus dem Blickwinkel der moderneren Makroökonomik findet sich bei Landmann (2001).
} 
Rückwirkungen auf die Inlands- und Auslandsnachfrage. Es ist in der Tat nicht schwierig, ein theoretisches Modell zu konstruieren, in dem der dargestellte Kaufkrafteffekt vorübergehend zum Tragen kommt, wenn die Überwälzungsprozesse erst mit Verzögerung einsetzen (Jerger 1999, Kap. 2.3). Eine andere Frage ist, ob diese Denkmöglichkeit empirische Relevanz besitzt. Nur auf der Basis von Daten kann man beurteilen, ob die Anpassungsverzögerungen groß genug sind, damit der Kaufkrafteffekt tatsächlich beschäftigungswirksam wird. Ein einschlägiger ökonometrischer Test mit Quartalsdaten ist negativ ausgefallen (Jerger 1999, Kap. 2.5).

Sowohl theoretisch als auch empirisch eindeutig nachweisbar ist demgegenüber, dass steigende Löhne selbst unter expliziter Berücksichtigung des Kaufkrafteffekts nachfrage- und beschäftigungsmindernd wirken, wenn die Überwälzung auf die Güterpreise erst einmal erfolgt ist. Denn der Anstieg des Preisniveaus schmälert nicht nur den realen Kaufkraftgewinn, der jedem einzelnen Beschäftigten durch eine gegebene nominale Lohnerhöhung zufällt, sondern senkt zudem die Güternachfrage auch durch seine Rückwirkungen auf den Kapitalmarktzins und/oder die internationale Wettbewerbsfähigkeit der Unternehmungen. Es lässt sich im Rahmen des aggregierten Angebots-Nachfrage-Modells leicht zeigen, dass der direkte Kaufkrafteffekt einer Nominallohnerhöhung durch die Sekundärwirkungen des steigenden Preisniveaus mehr als wettgemacht wird und daher als Beschäftigungswirkung das Gegenteil dessen eintreten muss, was Abbildung 3 suggeriert. ${ }^{1.5}$ Mehr noch, es kann sogar zu einem eigentlichen Kaufkraftparadoxon des Lohnes kommen; und zwar dann, wenn eine Nominallohnerhöhung das Preisniveausso stark anhebt und die Beschäftigung so stark senkt, dass die reale Lohnsumme zurückgeht. ${ }^{16}$ In diesem Fall verfügen die Arbeitnehmer insgesamt am Ende sogar über weniger Kaufkraft als vor der Lohnerhöhung. Dies wiederum bedeutet, dass die negative Beschäftigungswirkung einer Lohnerhöhung durch den Kaufkrafteffekt verstärkt wird - womit das ursprüngliche Kaufkraftargument geradezu auf den Kopf gestellt wird.

\section{Zusammenfassung und Schlussfolgerungen}

Die wesentlichen Punkte unseres Arguments lassen sich wie folgt zusammenfassen:

1. Die Debatte über die Beschäftigungswirkungen der Lohnpolitik sollte in Kategorien des Nominallohns geführt werden. Denn dies ist die Variable, die in Tarifvertragsverhandlungen zur Disposition steht. Der Reallohn bildet sich demgegenüber endogen und reflektiert neben den Anpassungsprozessen, die die Nominallohnpolitik auslöst, auch weitere exogene Einflüsse. Daher ist der Reallohn - ob absolut oder in Relation zur Arbeitsproduktivität - kein tauglicher Indikator für das Verhalten der Lohnpolitik.

\footnotetext{
${ }^{15}$ Formal läuft das Argument darauf hinaus, dass die Lage der Nachfragefunktion aus Abbildung 1 zusätzlich von der realen Lohnsumme abhängig wird. Dies hat zur Folge, dass eine Nominallohnerhöhung nicht nur die Angebotskurve nach oben verschiebt, sondern auch die Nachfragekurve - letztere allerdings in geringerem Ausmaß (Jerger/Landmann 2001). Eine Modellierung des Kaufkrafteffekts mit stringenter Mikrofundierung der Nachfrageseite findet sich bei Jerger/Michaelis (2000).

${ }^{16}$ Vgl. Jerger/Landmann (2001). Es lässt sich zeigen, dass das Auftreten des Kaufkraftparadoxons von der Substitutionselastizität zwischen Kapital und Arbeit abhängt.
} 
2. Das geeignetste Denkinstrument zur Analyse der Beschäftigungswirkungen der Nominallohnpolitik, insbesondere im Vergleich zu den Wirkungen der Nachfragepolitik (Geldund Fiskalpolitik), ist ein Standard-Paradigma der makroökonomischen Lehrbücher, nämlich das aggregierte Angebots-Nachfrage-Modell. Nach diesem Modell hängt die Beschäftigung davon ab, wie sich die Lohnpolitik relativ zur Nachfragepolitik verhält. Empirisch gestützt wird diese Aussage durch die Beobachtung, dass die Beschäftigung sehr eng mit dem Verhältnis zwischen nominaler Gesamtnachfrage und Nominallohn korreliert ist.

3. Hieraus folgt unmittelbar, dass die Orientierung der Lohnpolitik am Produktivitätswachstum für die Beschäftigung allein noch gar nichts präjudiziert. Eine mittelbare Bedeutung für die Beschäftigung erhält die Produktivitätsregel erst dadurch, dass sie den Expansionsspielraum definiert, den eine auf die Erhaltung der Preisniveaustabilität verpflichtete Nachfragepolitik ausschöpfen kann, ohne ihren Stabilitätsauftrag zu verletzen.

4. Das Kaufkraftargument - also das Argument, dass Löhne nicht nur Kosten, sondern auch Kaufkraft bedeuten - ändert an den geschilderten Zusammenhängen zwischen Löhnen, Nachfrage, Preisniveau und Beschäftigung nichts grundsätzliches. Insbesondere lässt sich mit diesem Argument die von den Gewerkschaften gerne vorgebrachte These, dass Lohnerhöhungen dank ihrem Kaufkrafteffekt die Beschäftigung beleben, nicht stützen und schon gar nicht lässt sich eine solche These aus dem theoretischen Gedankengebäude von Keynes ableiten.

Man kann nicht behaupten, dass irgendeine dieser Aussagen den Konsumenten eines marktgängigen Lehrbuchs der Makroökonomik erstaunen könnte. Erstaunlich ist höchstens, wie sich an Fragen, die mit einfachen Instrumenten der makroökonomischen Analyse beantwortbar sind, immer wieder hitzige Debatten entzünden. Jenseits der simplen Tatsache, dass nicht alle Einsichten der Makroökonomik allen Partikularinteressen gleichermaßen ins Konzept passen können, gibt es vor allem einen Aspekt, der offenbar immer wieder für Konfusion sorgt: Die Erklärung der Beschäftigung und ihrer Veränderungen kann auf ganz unterschiedlichen Ebenen ansetzen (Landmann/Jerger 1999, Kap. 8). Die Argumentation mit dem aggregierten Angebots-Nachfrage-Modell bewegt sich insofern auf einer sehr elementaren Ebene, als dieses Modell zwar die Wirkungen der Nominallohnpolitik und der Nachfragepolitik beschreibt, deren Verhalten selbst aber nicht analysiert. Einen Schritt weiter gehen Ansätze, welche die Lohnpolitik und/oder die Nachfragepolitik ihrerseits mit Hilfe von Reaktionsfunktionen endogenisieren. Eine solche erweiterte Analyse kann zu zusätzlichen Einsichten führen, und bisweilen mag es dann so scheinen, als würden diese weiterreichenden Einsichten das elementarere Wirkungsmodell entkräften. Aber der Schein trügt.

Ein Beispiel: Wenn die Mehrheit des Sachverständigenrates die eingangs zitierte Meinung vertritt, dass eine Besserung der Konjunktur "keine übermäßigen" Beschäftigungsgewinne bringe, so tut sie dies unter Berufung auf Berechnungen der OECD, aus denen hervorgeht, dass die Arbeitslosigkeit in Deutschland überwiegend strukturell und nicht konjunkturell bedingt sei. Solche Berechnungen stützen sich auf Modelle, die davon ausgehen, dass eine akzelerierende Lohn-Preis-Spirale einsetzt, wenn die Arbeitslosenquote ein bestimmtes - eben "strukturelles" - Niveau unterschreitet. Hieraus folgt, wie wir seit Phelps (1967) und Friedman (1968) wissen, dass die Nachfragepolitik die Arbeitslosenquote nicht dauerhaft unter diesem "strukturellen" Niveau halten kann, weil sie durch die induzierten Nominallohnerhöhungen immer wieder konterkariert wird. Aber die hierdurch begründete Skepsis bezüglich 
der Möglichkeiten der Nachfragepolitik steht nicht im Widerspruch zu der symmetrischen Rolle, die das Angebots-Nachfrage-Modell der Lohnpolitik und der Nachfragepolitik bei der Erklärung der Beschäftigung zuweist. Vielmehr bezieht sich die Skepsis darauf, ob die Lohnpolitik willens ist, sich der Disziplin zu unterwerfen, die eine Vollbeschäftigungsstrategie gemäß dem Angebots-Nachfrage-Modell verlangt, wenn gleichzeitig das Ziel der Preisniveaustabilität im Auge behalten werden soll.

Wie diese Disziplin hergestellt werden kann, ist letztlich das Problem, das gelöst werden muss, wenn die strukturelle Arbeitslosenquote sinken soll. Im Blickpunkt stehen dabei inflexible institutionelle Regelwerke, beschäftigungsfeindliche Anreize in den Transfer- und Steuersystemen, marktwidrige Lohnstrukturen und andere Funktionsstörungen auf den Arbeits- und Gütermärkten, die zu korrigieren sind. Auf dieser Ebene, auf der es darum geht, wie die Spielregeln und Rahmenbedingungen des Arbeitsmarktes reformiert werden können, damit die Lohnpolitik die notwendigen Anreize erhält, ihren Beitrag zu einer nachhaltigen Verbesserung der Beschäftigungslage zu leisten, mögen noch viele Fragen klärungsbedürftig sein. Aber die vorgelagerte Frage, worin dieser Beitrag überhaupt besteht, und wie die Lohnpolitik hierbei mit der Nachfragepolitik zusammenwirken muss, ist - auch wenn die wiederkehrenden Debatten immer wieder das Gegenteil suggerieren - nicht mehr klärungsbedürftig. Die Antworten gehören längst zur Routine jedes volkswirtschaftlichen Grundstudiums.

\section{Literatur}

Bernanke, Ben S. (1986): Alternative explanations of the money-income correlation, in: CarnegieRochester Conference Series on Public Policy, Vol. 25, p. 49-100

Blanchard, Olivier J. (1997): The Medium Run, in: Brookings Papers on Economic Activity, 2, S. $89-141$

Blanchard, Olivier J. (2000a): Macroeconomics, 2. Auflage, Prentice Hall

Blanchard, Olivier J. (2000b): The Economics of Unemployment - Shocks, Institutions, and Interactions, Lionel Robbins Lectures, London School of Economics

Blanchard, Olivier J., Francesco Giavazzi (2001): Macroeconomic effects of regulation and deregulation in goods and labor markets, NBER Working Paper No. 8120

Blanchard, Olivier J., Danny Quah (1989): The dynamic effects of aggregate demand and supply disturbances, in: American Economic Review, Vol. 79, p. 655-673

Caballero, Ricardo J., Mohamad L. Hammour (1998): Jobless growth: appropriability, factor substitution, and unemployment, in: Carnegie-Rochester Conference Series on Public Policy, Vol. 48, p. 51-94

Friedman, Milton (1968): The role of monetary policy, in: American Economic Review, Vol. 58, p. $1-17$

Gordon, Robert J. (2000): Macroeconomics, 8. Auflage, Addison-Wesley

Heise, Arne, Th. Schulten (1999): Lohndumping in der EWU - geht ein Gespenst um in Europa? eine Replik, in: Wirtschaftsdienst, Heft 2, S. 89-94

Jensen, Henrik (1999): Targeting Nominal Income Growth or Inflation? CEPR Discussion Paper No. 2341, Dezember

Jerger, Jürgen (1999): Nachfragesteuerung, Lohnbildung und Beschäftigung, Tübingen

Jerger, Jürgen, Oliver Landmann (2001): Lohnhöhe, Güternachfrage und Beschäftigung, in: WiSt Wirtschaftswissenschaftliches Studium, Heft 3, S. 143-149 
Jerger, Jürgen, Jochen Michaelis (2000): Nominal Wages, Real Demand and Aggregate Employment, Beiträge zur wirtschaftspolitischen Forschung der Universität Erlangen-Nürnberg Nr. 10

Kalecki, Michal (1971): Class struggle and distribution of national income, in: Kalecki, Michal: Selected essays in the dynamics of the capitalist economy, Cambridge University Press, 1971, p. 156-164

Keynes, John Maynard (1936): The general theory of employment, interest and money, in: Moggridge, Donald (ed.): The collected writings of John Maynard Keynes, Vol. VII, Macmillan and Cambridge University Press, 1989

Kromphardt, Jürgen (1999): Lohnsenkungswettbewerb in der EWU - Deflationsgefahr oder Beschäftigungsimpuls?, in: Wirtschaftsdienst, Heft 2, S. 85-89

Landmann, Oliver (2001): Wage Restraint, Employment, and the Legacy of the General Theory's Chapter 19, in: S. Berninghaus (Hrsg.), ..., Springer Verlag, Berlin/Heidelberg [im Erscheinen]

Landmann, Oliver, Jürgen Jerger (1993): Unemployment and the real wage gap: A reappraisal of the German experience, in: Weltwirtschaftliches Archiv, Vol. 129, p. 689-717

Landmann, Oliver, Jürgen Jerger (1999): Beschäftigungstheorie, Heidelberg, Berlin: SpringerVerlag

Lehment, Harmen (1999): Zur Bestimmung des beschäftigungsneutralen Lohnerhöhungsspielraums, in: Die Weltwirtschaft (1), S. 79-89.

Lerner, Abba (1951): The Economics of Employment, New York

Hall, Robert E., N. Gregory Mankiw (1994): Nominal income targeting, in: Mankiw, N. Gregory (ed.): Monetary policy, University of Chicago Press, p. 71-94

Marschak, Jacob (1927): Hohe Löhne und die Wirtschaft, in: Die Arbeit, 4

Phelps, Edmund S. (1967): Phillips curves, expectations of inflation and optimal unemployment over time, in: Economica, Vol. 34, p. 254-281

Sachverständigenrat zur Begutachtung der gesamtwirtschaftlichen Entwicklung (1999): Wirtschaftspolitik unter Reformdruck (Jahresgutachten 1999/00), Bundestagsdrucksache $14 / 2223$

Solow, Robert S. (1986): Unemployment: Getting the Questions Right, in: Economica, Vol. 53 (Supplement), S. S23-S34

von Weizsäcker, C. Christian (2000): Über die Schlusspassage der General Theory - Gedanken zum Einfluss ökonomischer Theorien auf die Politik, in: Perspektiven der Wirtschaftspolitik, Band 1, Heft 1, S. 35-52

Taylor, John B. (1999): Monetary Policy Rules, University of Chicago Press

Whitley, John (1994): A course in macroeconomic modelling and forecasting, New York: Harvester Wheatsheaf 1994 


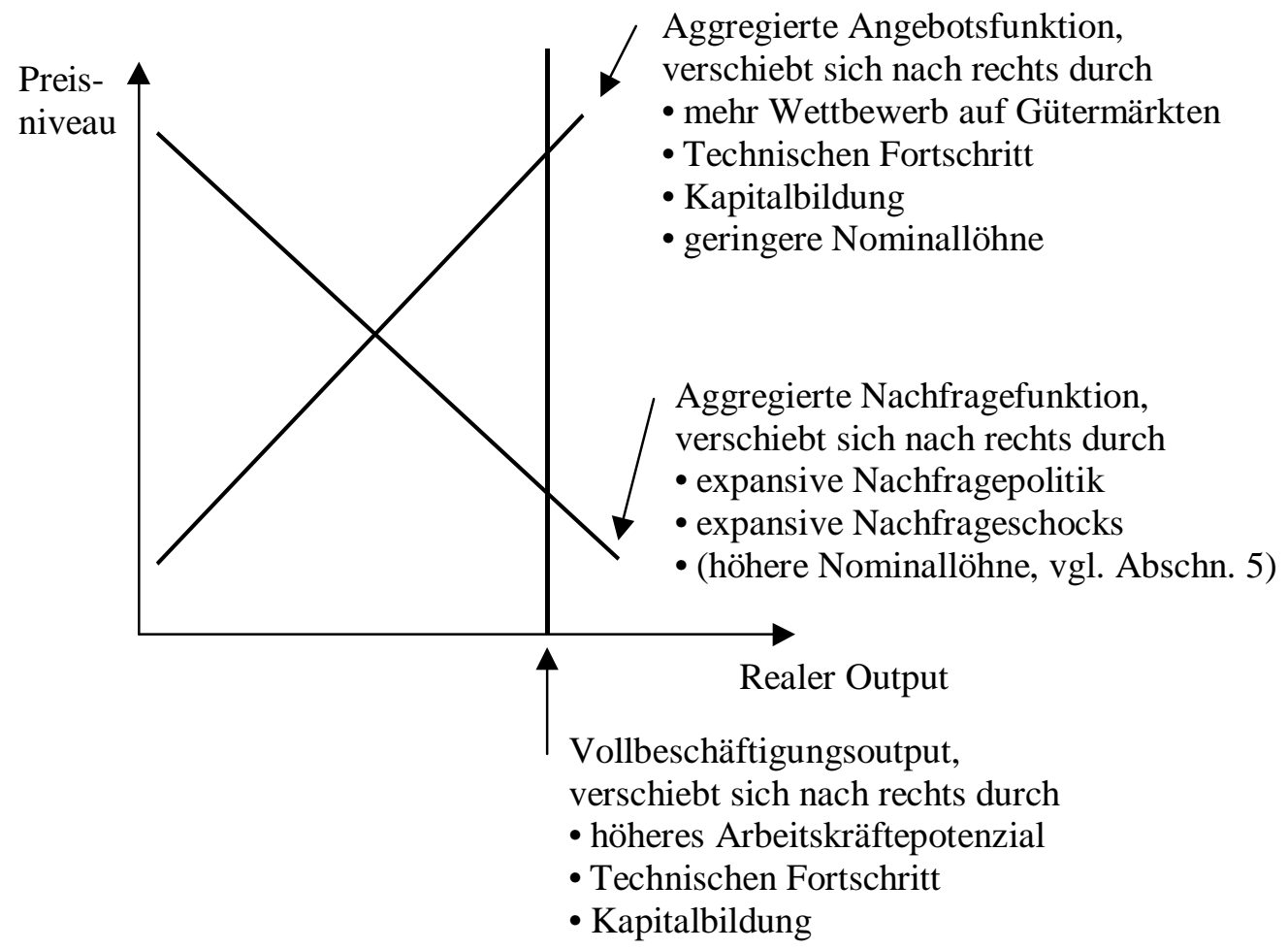

Abbildung 1: Das makroökonomische Angebots-Nachfrage-Modell 

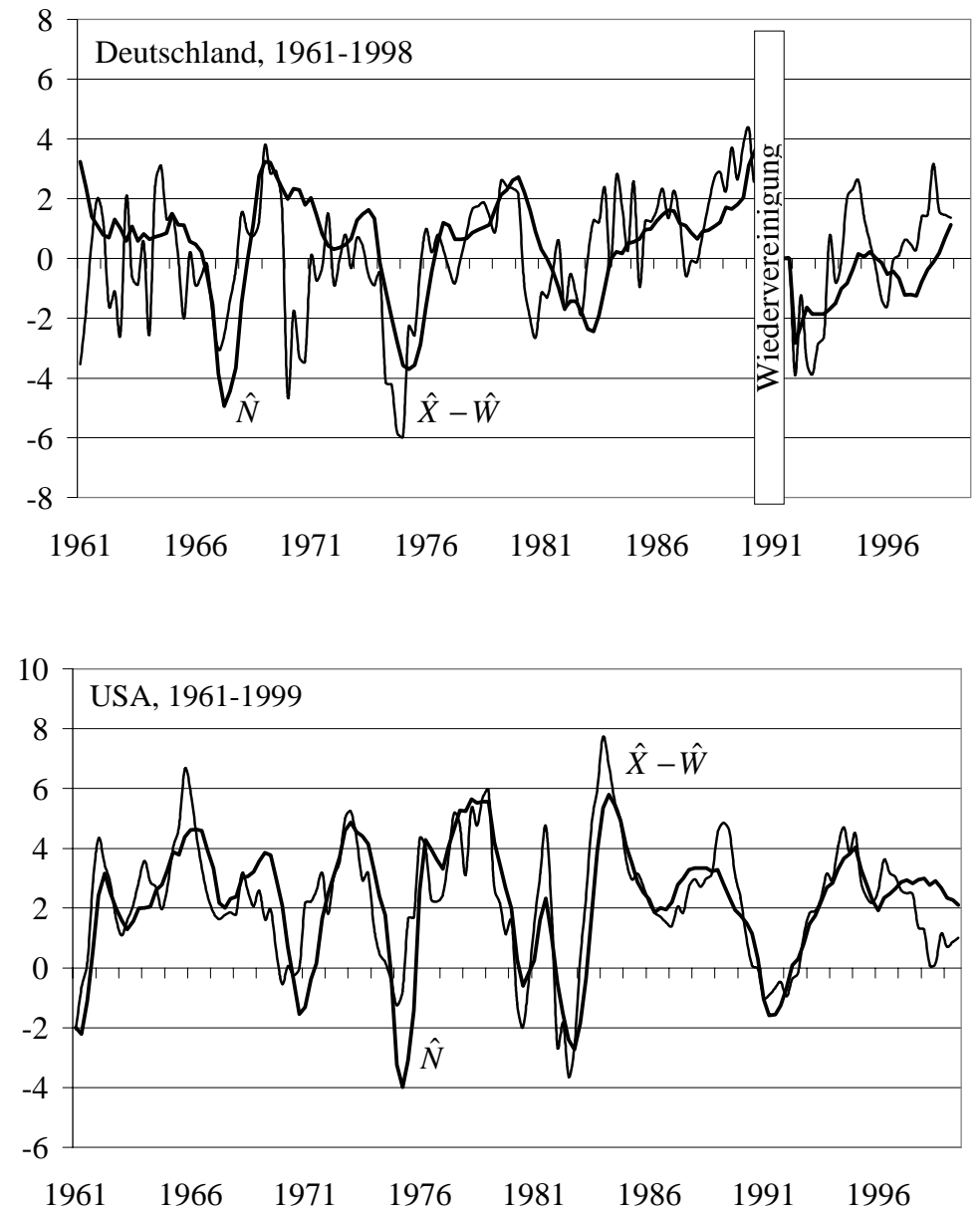

Abbildung 2: Das makroökonomische Angebots-Nachfragemodell auf dem empirischen Prüfstand; Quelle: OECD, eigene Berechnungen
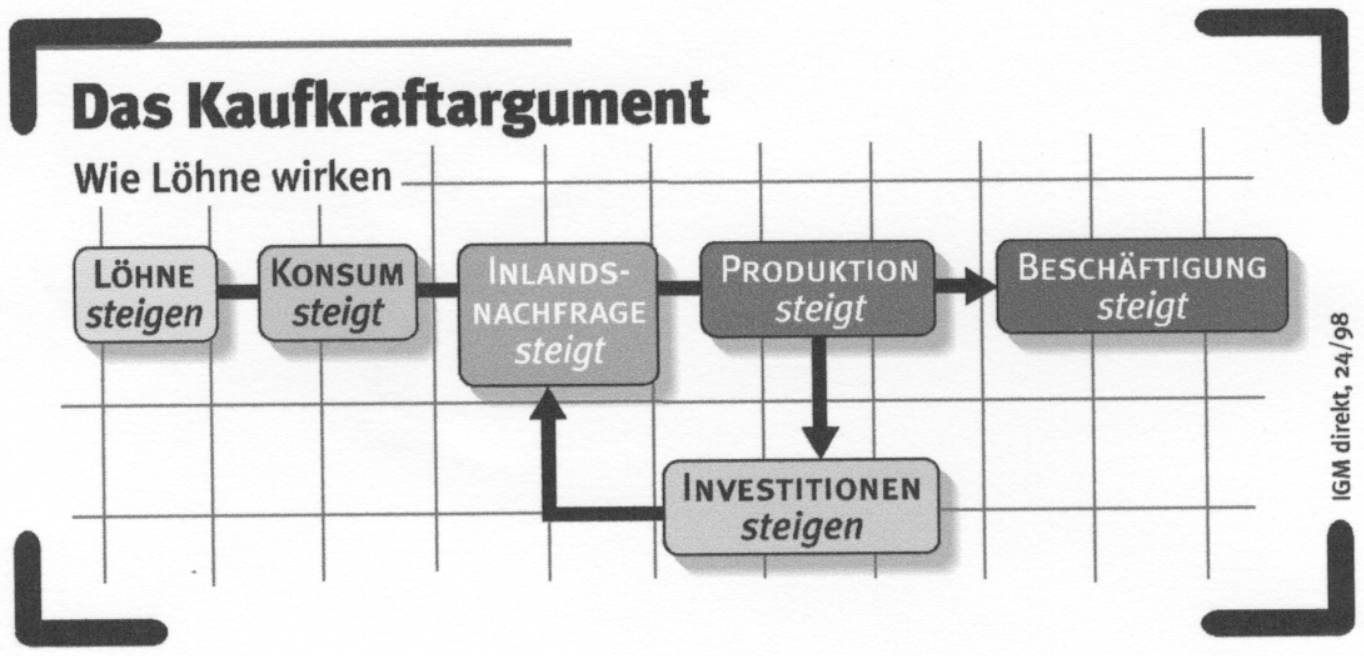

Abbildung 3: Das Kaufkraftargument der Löhne (Darstellung der IG Metall) 